\title{
Increased Neutralizing Antibody Response after Simultaneous Immunization with Leucogen and the Feline Leukemia Virus Transmembrane Protein
}

\author{
Stefan Langhammer Uwe Fiebig Reinhard Kurth Joachim Denner
}

Robert Koch Institute, Berlin, Germany

\section{Key Words}

Feline leukemia virus $\cdot$ Vaccination $\cdot$ Neutralizing

antibodies $\cdot$ Transmembrane envelope protein

\begin{abstract}
To develop improved vaccination strategies against feline leukemia virus (FeLV), rats were immunized with the transmembrane envelope protein p15E of FeLV alone or in combination with the commercial vaccine Leucogen ${ }^{\circledR}$ comprising the nonglycosylated FeLV surface envelope protein. Binding and neutralizing antibodies were induced in both groups and in the group immunized with Leucogen alone. Higher titers of antibodies neutralizing FeLV were induced by simultaneous immunization with Leucogen and p15E compared to the responses using Leucogen or p15E alone, suggesting that combination vaccines should be used in the future. Epitope mapping of p15E-specific antibodies induced by simultaneous immunization with Leucogen and p15E revealed the same pattern of response as obtained after immunization with p15E alone: one epitope was localized in the membrane-proximal external region (MPER) and the other in the fusion peptide-proximal region, and they are related to the epitopes detected after immunization with p15E of the porcine endogenous retrovirus and the koala retrovirus. The data indicate that these epitopes in the MPER are an effective target for neutralization and that antigens containing them may therefore prove to be a useful component of vaccines against retroviruses, including HIV-1.
\end{abstract}

Copyright $\odot 2010$ S. Karger AG, Basel

\section{KARGER}

Fax +4161306 1234 E-Mail karger@karger.ch www.karger.com

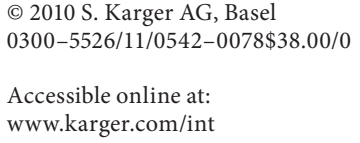

\section{Introduction}

Immunization is the most effective method to prevent diseases caused by infectious agents. The success of a variety of vaccines against diseases such as smallpox, measles, mumps, and poliomyelitis has dramatically reduced morbidity and mortality worldwide and has led to the eradication of smallpox [1]. It is of interest that most, if not all, successful vaccines are based on the induction of neutralizing antibodies. Although the development of vaccines against human immunodeficiency virus-1 (HIV-1), the retrovirus that causes AIDS, is one of the major tasks of present vaccine development, all attempts up to now have failed [2]. In addition, passive immunization using broadly neutralizing monoclonal antibodies such as 2F5 and 4E10 derived from HIV-infected patients has been used to treat HIV infection [3]. The epitopes recognized by monoclonal antibodies 2F5 and 4E10 are located in the membrane-proximal external region (MPER) of gp41 $[4,5]$. Numerous attempts to induce broadly neutralizing antibodies such as 2F5 and 4E10 using recombinant gp41 or synthetic peptides have failed [6], although, recently, oral immunization with bovine papilloma virus/HIV-1 gp41 chimeric virus-like particles induced very low titers of neutralizing antibodies in mice [7].

In contrast, vaccines against feline leukemia virus (FeLV) are commercially available, showing that antiretroviral vaccines are not impossible. FeLV is a gammaretrovirus comprising 3 subtypes, i.e. A, B and C [8], of 
which FeLV-A is the predominant serotype in cats [9]. FeLV induces fatal leukemia, lymphomas, and immunosuppression associated with opportunistic infections in infected cats. The number of cases of FeLV-induced disease has been reduced by the development and use of several vaccines against FeLV-A. However, none of the 7 commercial FeLV vaccines currently available in the USA and Europe provide 100\% protection against infection. Three vaccines are composed of inactivated whole virus, 2 are gp70 subunit vaccines, and 2 are recombinant vaccines [10]. The Leucogen ${ }^{\circledR}$ vaccine contains the recombinant nonglycosylated $\mathrm{p} 45$ of the surface envelope glycoprotein gp70 and is one of the most effective [11].

Recently, we induced neutralizing antibodies specific for the transmembrane envelope protein $\mathrm{p} 15 \mathrm{E}$ of porcine endogenous retrovirus (PERV) [12], FeLV-A [13, 14], and koala retrovirus (KoRV) [15] in goats, rats, and cats. The epitopes recognized by the immune sera were located in 2 distinct regions of the transmembrane envelope protein including the MPER, and a sequence homology between the epitopes in the MPER of FeLV, KoRV, PERV, and HIV1 was reported [12]. Our data suggest that these regions of the transmembrane envelope protein represent a vulnerable target for neutralization. Therefore, this antigen may prove to be a useful component of all antiretroviral vaccines.

Here we describe for the first time the induction of higher titers of neutralizing antibodies in rats by combined immunization with the surface envelope protein in the form of the commercially available Leucogen vaccine and the recombinant ectodomain of p15E of FeLV, and we describe the localization of the epitopes recognized. We show that a vaccine containing both antigens is more powerful compared to each single antigen. These findings are of great importance for the general improvement of retroviral vaccines.

\section{Materials and Methods}

Experimental Animals, Antigens, and Immunization

Eighteen Wistar rats (Charles River, Wilmington, Mass., USA) were immunized twice intramuscularly and subcutaneously at weeks 0 and 3 . Leucogen, containing $0.1 \mathrm{mg}$ p 45 plus Quil-A and aluminium hydroxide as adjuvants (Virbac; lot No. 80986902143521 ), was given alone or mixed with $100 \mu \mathrm{g}$ p15E. Immunization with 100 or $500 \mu \mathrm{g}$ p15E alone was performed at a dilution of 7:3 in Montanide ISA 720 (Seppic, France; lot No. 143521). The recombinant ectodomain of p15E of FeLV-A used for immunization was prepared as described [13]. Briefly, the ectodomain (aa 476-583) of FeLV-A p15E was cloned into the pCal-n vector (Stratagene Europe, Amsterdam, The Netherlands) and expressed in Esterichia coli BL21 DE3 cells. The p15E N-terminally fused to a 4 -kDa calmodulin-binding protein was purified by affinity chromatography.

\section{Quantification of Antigen-Specific Immunoglobulins by}

\section{ELISA}

FeLV-A p15E and Leucogen p45-specific antibody titers were determined by ELISA. Briefly, ELISA plates were coated for $1 \mathrm{~h}$ at $37^{\circ}$ with affinity-purified recombinant p15E protein diluted in $\mathrm{PBS}$ or for $18 \mathrm{~h}$ at $37^{\circ}$ with Leucogen $\mathrm{p} 45$ vaccine component diluted in PBS (100 ng/well). The ELISA plates were then washed with PBS containing $0.1 \%$ Tween 20 and blocked for $1 \mathrm{~h}$ at room temperature with PBS containing 0.1\% Tween 20 and 5\% BSA. Serum samples, diluted with PBS containing 2.5\% BSA and $0.1 \%$ Tween 20, were added to the ELISA plates at a starting dilution of $1: 1,000$, diluted further (4-fold dilution series), and incubated for $1 \mathrm{~h}$ at $37^{\circ}$. The ELISA plates were then washed 3 times with PBS containing $0.1 \%$ Tween 20 . A horseradish peroxidase-conjugated secondary antibody specific for rat IgG diluted 1:3,500 with PBS containing $2.5 \%$ BSA and $0.1 \%$ Tween 20 was used to detect antigen-specific immunoglobulin. Incubation for $1 \mathrm{~h}$ at $37^{\circ}$ was followed by 5 washings with PBS containing $0.1 \%$ Tween 20 . Finally, the ELISA plates were developed by addition of OPD (o-phenylenediamine dihydrochloride) diluted in PBS (50 $\mu \mathrm{g} /$ well) plus $0.1 \% \mathrm{H}_{2} \mathrm{O}_{2}$ followed by inactivation with $30 \mu \mathrm{H}_{2} \mathrm{SO}_{4}(5 \mathrm{~N})$ after $10 \mathrm{~min}$. Protein-specific antibody endpoint titers are reported as the dilution giving an $\mathrm{OD}_{492 / 620 \mathrm{~nm}}$ reading above that of preimmune sera.

\section{Virus Neutralization Assay}

The virus stock for the neutralization assay was prepared as cell-free supernatant from feline embryonic fibroblast (FEA) cells infected with the FeLV-A Glasgow strain (kindly provided by M. Reinacher, Giessen, Germany and O. Jarrett, Glasgow, UK). The stock was titrated on uninfected FEA cells and was shown to have a titer of $10^{4.76} \mathrm{TCID}_{50} / \mathrm{ml}$. Neutralization assays were performed as follows: 1 day before the assay, 6,000 uninfected FEA cells per well were seeded into 96-well microtiter plates. Preimmune and immune sera were heat-inactivated at $56^{\circ}$ for $30 \mathrm{~min}$. $50 \mu \mathrm{l}$ of stock virus were incubated with 4 -fold serial dilutions of serum for $30 \mathrm{~min}$ at $37^{\circ}$ and then transferred to the cells. After 3 days of incubation, cells were freeze-thawed 3 times and a lysis buffer containing $20 \mathrm{mg} / \mathrm{ml}$ of proteinase $\mathrm{K}$ in PCR buffer $(50 \mathrm{mM} \mathrm{KCl}$, $1.5 \mathrm{mM} \mathrm{MgCl}_{2}$, and $10 \mathrm{~mm}$ Tris- $\mathrm{HCl} ; \mathrm{pH} 8.4$ ) was added. The cells were incubated for $3 \mathrm{~h}$ at $56^{\circ}$ followed by $10 \mathrm{~min}$ at $95^{\circ}$ to inhibit proteinase K activity. Proviral DNA was measured by real-time PCR as described below. To determine the titer of neutralizing antibodies, the dilution with provirus integration less than $100 \%$ was determined.

\section{Real-Time PCR}

An internal probe FAM-5'-TTAAGCACCTGGGCCCCGGC3'-DQ (Eurogentec, Cologne, Germany) was used together with FeLV-specific primers. The sense primer 5'-TCAAGTATGTTC-

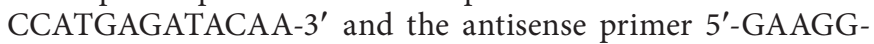
TCGAACTCTGGTCAACT-3' were used to amplify and to quantify a 185-bp product from the exogenous U3 sequence in the LTR region of the FeLV-A provirus genome. The $25-\mu 1$ reaction mixture consisted of $1 \times$ PCR buffer with $1 \mathrm{mM} \mathrm{MgCl}_{2} ; 0.5 \mu \mathrm{M}$ each 
of dATP, dCTP, dGTP, and dTTP; 5 pmol of each primer; 5 pmol of probe; $1.25 \mathrm{U}$ Amplitaq Gold polymerase, and $2 \mu \mathrm{l}$ lysis mixture. The thermal cycling conditions used were $12 \mathrm{~min}$ at $95^{\circ}$ followed by 50 cycles of $1 \mathrm{~min}$ at $95^{\circ}, 1 \mathrm{~min}$ at $59^{\circ}$ and $30 \mathrm{~s}$ at $72^{\circ}$ in a Stratagene MX4000 system.

\section{Epitope Mapping}

The entire p15E of the FeLV-A Glasgow strain was synthesized as a cellulose-adsorbed peptide spot library of 15-mer peptides overlapping by 13 amino acids (Jerini Biotools, Berlin, Germany) using a standard protocol. Sera were diluted 1:1,000 and incubated with the membrane for $3 \mathrm{~h}$, washed 3 times for $15 \mathrm{~min}$ with Tris-buffered saline (pH 7.5) containing 0.05\% Tween 20 (Sigma-Aldrich, Steinheim, Germany) and incubated for $2 \mathrm{~h}$ with a peroxidase-conjugated secondary antibody diluted 1:10,000. Binding was detected using a chemiluminescence detection solution (ECL, Amersham Pharmacia Biotech).

\section{Results}

The Transmembrane Envelope Protein p15E of Different FeLV Is Highly Conserved

The transmembrane envelope proteins of retroviruses are very similar in their structure due to their specific functions during infection. They are associated with conformational changes including an intramolecular interaction between the $\mathrm{N}$-terminal helix and the C-terminal helix [16]. Although the surface envelope protein gp70 of gammaretroviruses is not as variable as the surface envelope protein gp120 of HIV-1, the transmembrane envelope proteins of all retroviruses are more highly conserved and therefore possibly represent a better antigen for vaccination. To show this, the amino acid sequences of gp70 and p15E from the FeLV-A strains Glasgow-1, Rickard, and Sarma and the FeLV-B strain Gardner-Arnstein were aligned and analyzed as a phylogenetic tree using the program MegAlign (part of the DNASTAR package). A comparison of the phylogenetic trees for p15E and gp70 showed a higher divergence for the gp70 proteins than for the p15E proteins (fig. 1a). After having immunized rats, goats, and cats with the transmembrane envelope protein p15E of FeLV $[18,19]$, binding and neutralizing antibodies were induced and epitopes designated E1a, E2a, E1b, and E2a were identified in the fusion peptide-proximal region (FPPR) and membrane-proximal external region (MPER) of p15E (fig. 1b). The amino acid sequences of these epitopes are $100 \%$ identical in the strains analyzed (fig. 1c). These data indicate that the transmembrane envelope protein, especially the MPER, is a highly conserved target for neutralization and may be used to induce antibodies neutralizing all FeLV strains.
Neutralizing Activity Correlated with the Recognition of Epitope E1b

In order to induce neutralizing antibodies, a group of 6 rats (animals 1.1-1.6) was immunized with the ectodomain of the transmembrane envelope protein p15E of FeLV-A. Two out of 6 antisera obtained after boost immunization were able to inhibit the infection of FEA cells by FeLV-A. Both antisera showed neutralization titers of 1:4 as determined by a 4 -fold serum dilution. The percentages of provirus integration for antisera 1.2 and 1.4 were reduced to 25 and $4 \%$, and the neutralizing capacities were 75 and 96\%, respectively (fig. 2a). All antisera from this animal group showed strong binding antibodies against the recombinant p15E protein in ELISA (fig. 2b). Their titers of binding antibodies were in a range between $2.56 \times 10^{5}$ and $4.1 \times 10^{7}$; however, they did not correlate with the neutralizing capacity of the sera. When epitope mapping was performed using linear 15-mer peptides (overlapping by 13 amino acids) corresponding to the entire p15E of FeLV-A, covalently bound by the Cterminus to a cellulose membrane, 2 main epitopes were identified, i.e. E1b and E2b (fig. 2c, d). This finding confirms previous results showing the induction of neutralizing antibodies and the identification of the same epitopes after the immunization of goats and rats with FeLV-A p15E [13]. Interestingly, all antisera immunized with FeLV-A p15E recognized the E2b epitope but only antisera with neutralizing activity also recognized the E1b epitope (fig. 2c, d; antisera 1.2 and 1.4).

\section{Immunization with Leucogen Induced Binding and \\ Neutralizing Antibodies against $p 45$ in Rats}

To analyze whether Leucogen, which is comprised of the recombinant unglycosylated envelope surface protein p45 of FeLV, induces, as expected, binding and neutralizing antibodies a group of 3 rats was immunized with 1 dose and boosted with a second dose of Leucogen. All 3 antisera obtained showed neutralization titers of 1:16. The neutralization capacity ranged from 80 to $100 \%$ at a serum dilution of 1:4 (fig. 3a). These data showed that the induced neutralizing capacity in vitro is much higher after immunization with Leucogen when compared to immunization with p15E (fig. 2a). Binding antibodies from all 3 sera showed titers of $6.4 \times 10^{4}$ against recombinant p45 as determined by ELISA (fig. 3b). Therefore, the titers of binding antibodies induced by immunization with p15E (fig. 2b) were significantly lower when compared with the titers against $\mathrm{p} 45$ obtained from immunization with Leucogen (fig 2a). 


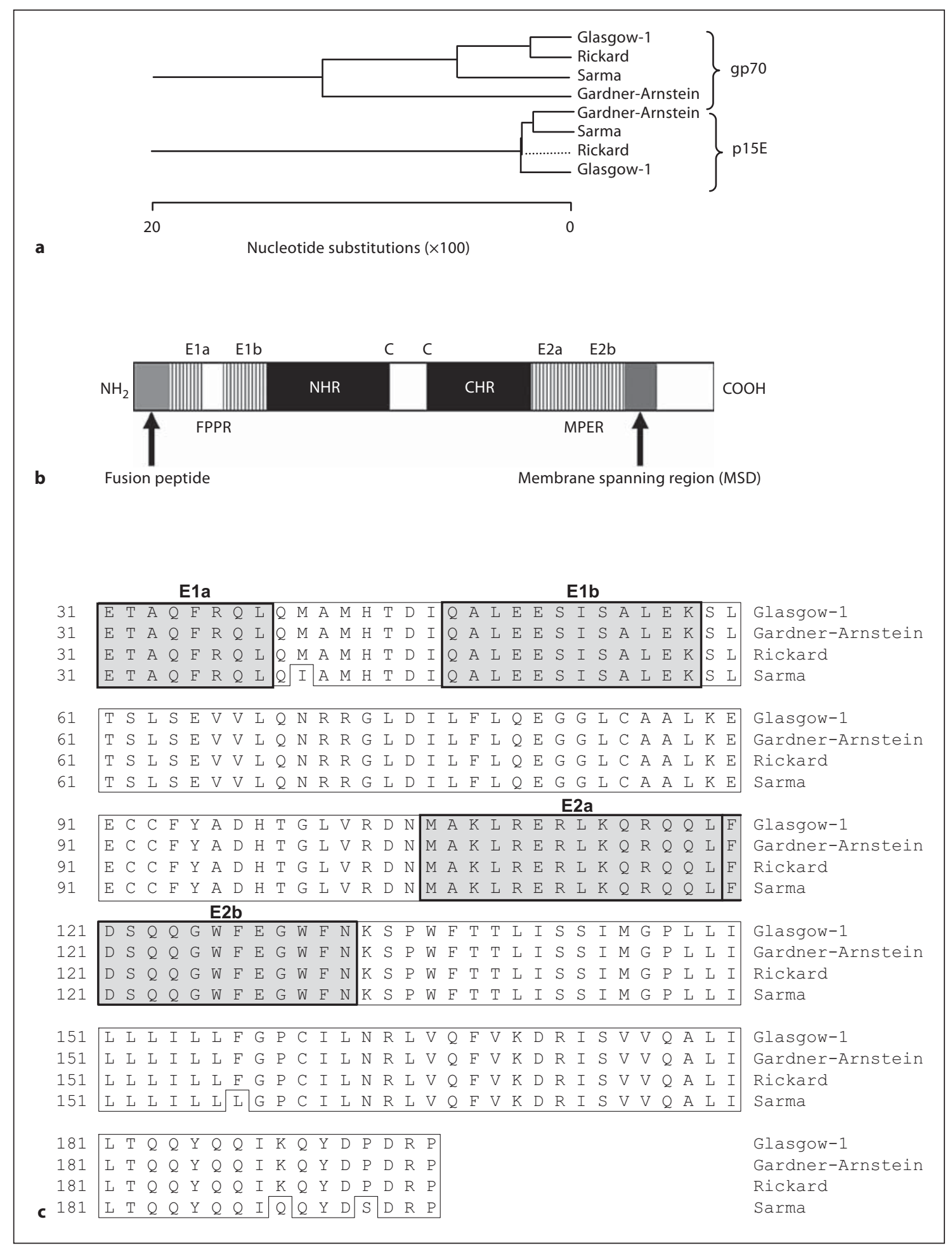

Fig. 1. a Comparison of p15E of different FeLV; unrooted phylogenetic tree of gp70 and of p15E of the FeLV strains Glasgow1, Rickard, Sarma (FeLV-A), and Gardner-Arnstein (FeLV-B). b Schematic presentation of the transmembrane envelope protein p15E of FeLV. NHR = N-Terminal helical region; $\mathrm{CHR}=\mathrm{C}$-terminal helical region. C-C cysteine loop, E1a, E1b, E2a, and E2b indi- cate the epitopes recognized by antisera induced by immunization with the ectodomain of p15E [13]. c Alignment of epitopes designated E1a, E2a, E1b, and E2a identified after the immunization of rats with the recombinant ectodomain of p15E of FeLV [13] (framed and indicated in grey) in different FeLV-A strains. 


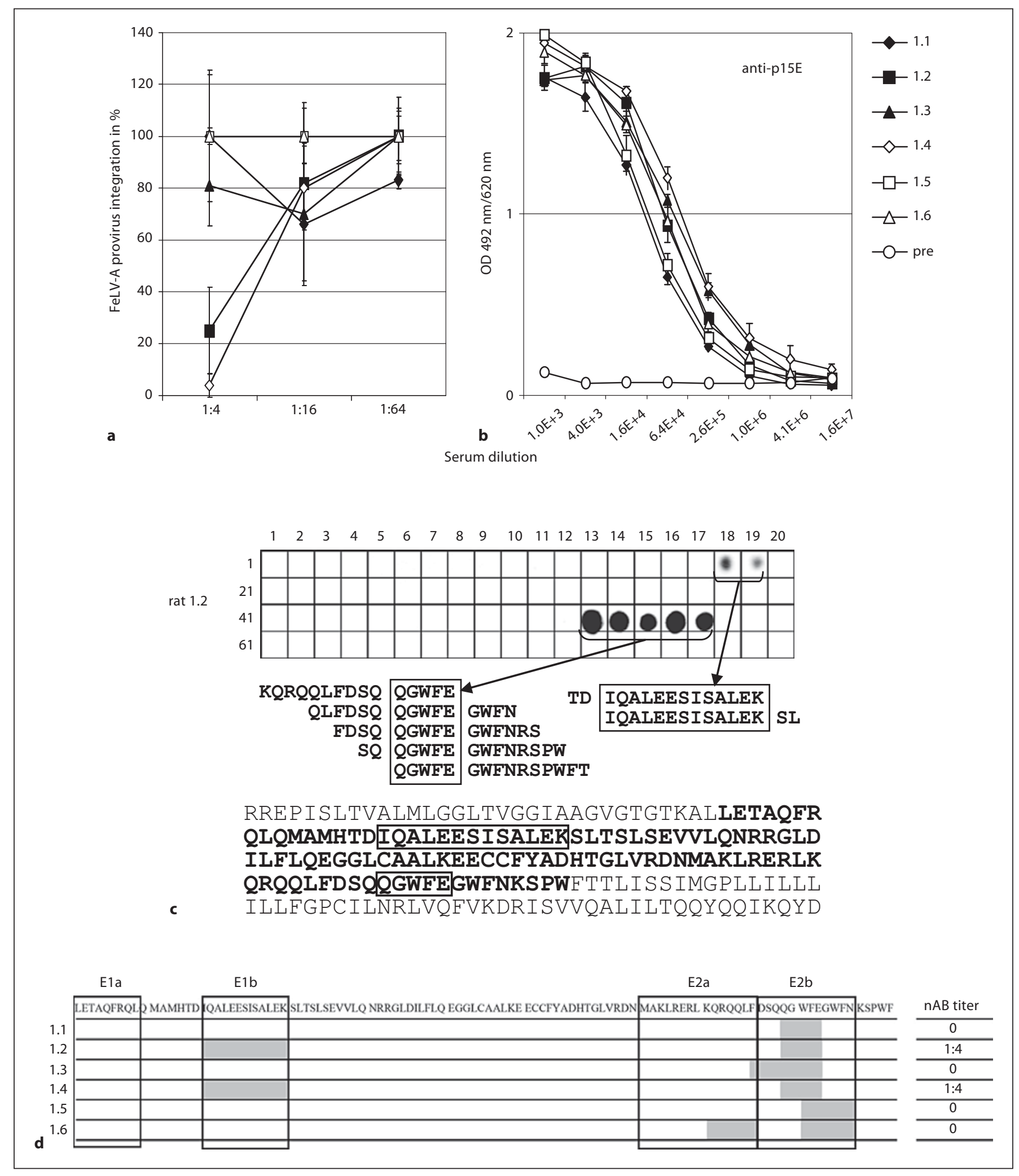

Fig. 2. Results of the immunization of rats with p15E of FeLV alone. a Neutralizing activity. b ELISA reactivity. c Epitope mapping of the serum from animal 1.2. d Schematic presentation of the epitopes of all rat antisera induced by immunizations with p15E alone. The titers of neutralizing antibodies are indicated on the right. 


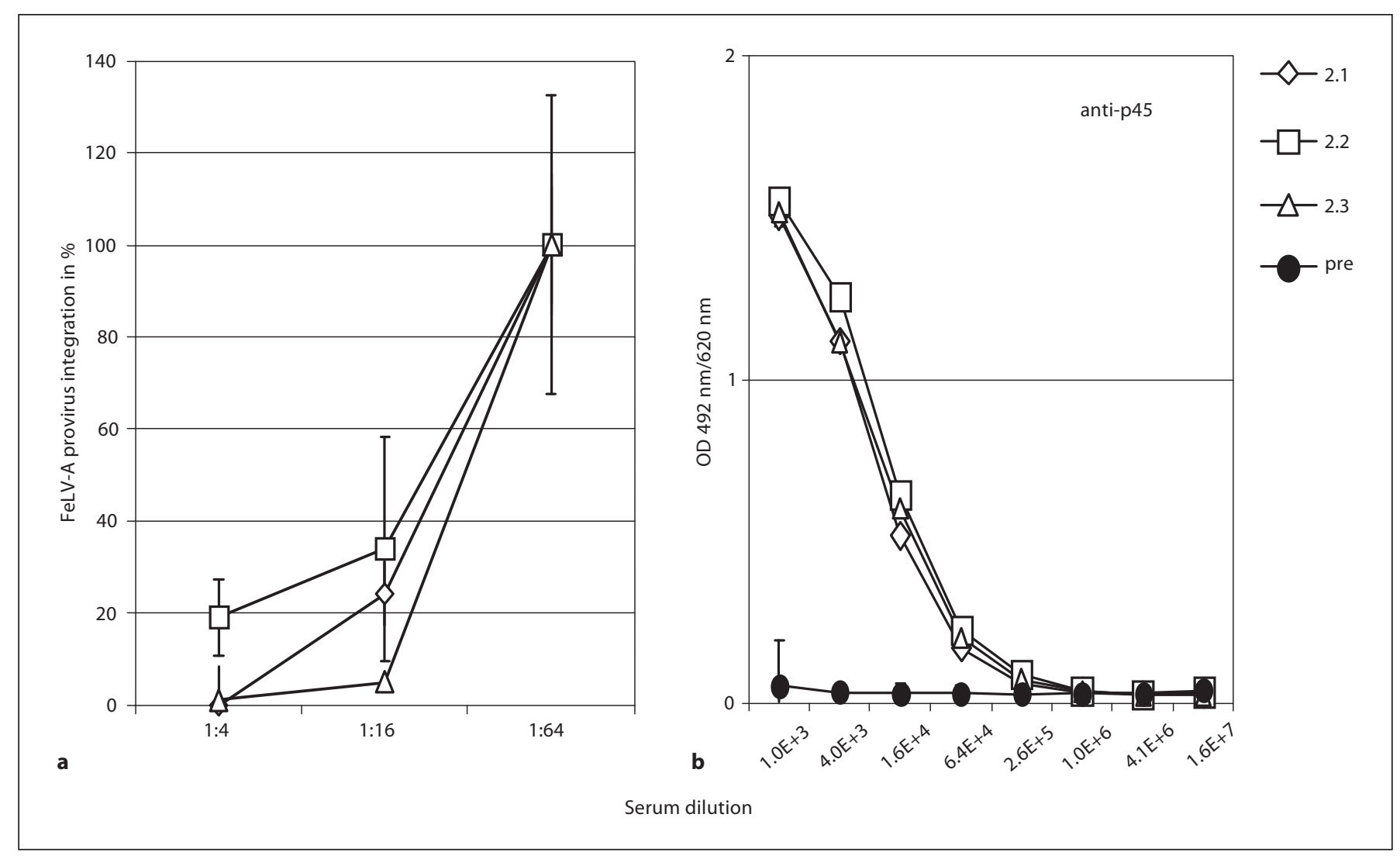

Fig. 3. Results of the immunization of rats with Leucogen. a Neutralizing activity. b ELISA reactivity against p45.

Combined Immunization with p15E and Leucogen Is

Superior to Immunization with Leucogen Alone

When the neutralizing capacity of the sera obtained after combined immunization with Leucogen and p15E (group 3) was compared with that of the sera obtained after immunization with p15E (group 1) or Leucogen alone (group 2), higher neutralization titers were observed in sera from group 3 (fig. 4a). All sera from rats immunized with the combination of Leucogen and p15E showed neutralizing activity against FeLV-A. Eight of 9 sera showed a neutralization titer $\geq 1: 64$, whereas only 1 serum (3.7) had a lower titer of 1:16 (fig. 4a). Five of 9 sera showed binding antibody titers against $\mathrm{p} 15 \mathrm{E}$ comparable to those obtained from immunizations with p15E alone (fig. 4b). The other 4 antisera showed lower antibody titers of $6.4 \times 10^{4}$ (fig. $4 \mathrm{~b}$; sera 3.5, 3.7, 3.8, and 3.9). A similar observation was made for antibody titers against p45: 5 out of 9 sera had titers comparable to those obtained from immunizations with Leucogen alone, but the other 4 showed lower titers of $1.6 \times 10^{4}$ (fig. $4 \mathrm{~b}$; antisera 3.4, 3.5, 3.7, and 3.8). These observations indicated that combined immunizations with p15E and Leucogen lead to reduced titers of binding antibodies on the one hand, but to an increased efficacy of neutralizing antibodies against FeLV on the other.

Epitope mapping of the sera from animals immunized with a combination of p15E and Leucogen detected all 4 epitopes (E1a, E1b, E2a, and E2b) as described previously [13]. The only epitope that all the antisera of this group recognized in common was the E1b epitope (fig. 4c). This epitope was also detected by both neutralizing sera obtained from immunizations with p15E alone (fig. 2d). Similar to the group immunized with p15E alone, nearly all sera obtained after combined immunizations of $\mathrm{p} 15 \mathrm{E}$ with Leucogen recognized the E2b epitope (fig. 4c). Only the serum from animal 3.7 failed to detect the E2b epitope. Interestingly, this antiserum not only showed the lowest titer of neutralizing antibodies (fig. 4a) but also reduced titers of binding antibodies against both p15E and p 45 (fig. 4b). Taken together, this data indicated that recognition of E1b and E2b seems to be associated with neutralizing activity. 


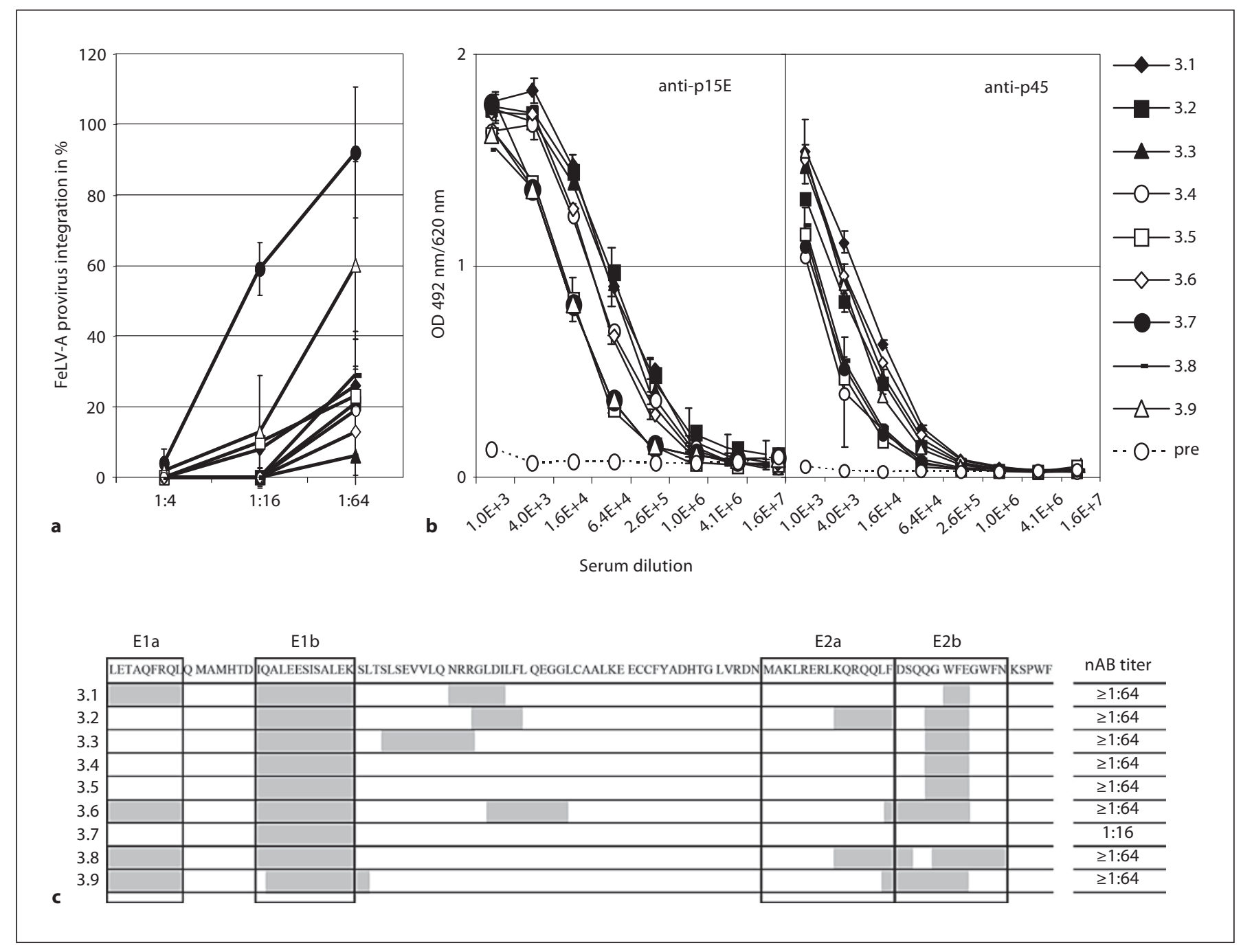

Fig. 4. Results of the combined immunization with $\mathrm{p} 15 \mathrm{E}$ and Leucogen. a Neutralizing activity. b ELISA reactivity against p45 and p15E. c Schematic presentation of the epitopes of all immune sera obtained by epitope mapping. The titers of neutralizing antibodies are indicated on the right.

\section{Discussion}

Immunizing rats with the transmembrane envelope protein $\mathrm{p} 15 \mathrm{E}$ of FeLV induced neutralizing antibodies which react with 2 epitopes: 1 located in the FPPR and the other in the MPER. This confirms previous results showing that similar neutralizing antibodies were also obtained in goats and rats [13]. Most importantly, higher titers of neutralizing antibodies were obtained when a combined immunization with $\mathrm{p} 15 \mathrm{E}$ and a licensed vaccine, i.e. Leucogen, was performed, indicating that this vaccine can be improved.
Vaccines against FeLV represent the first example of successful vaccines preventing a retroviral disease. Three types of FeLV vaccines are currently available: inactivated whole virus preparations, inactivated mixed subunit preparations from FeLV-infected tissue culture filtrate, and recombinant FeLV proteins. The commercially available vaccines containing inactivated FeLV subunit preparations are Fevaxyn, Leucocine, and Leukocell2. Leucogen is an example of a recombinant vaccine, and comprises recombinant nonglycosylated surface envelope protein p45. For all commercially available FeLV vaccines, there is only limited scientific data concerning the 
long-term duration of immunity after vaccination and none of the studies demonstrate $100 \%$ protection $[17,18]$. Therefore, annual revaccination is recommended. None of the vaccines regularly showed neutralizing antibodies; such antibodies are usually detected only after recovery from challenge [17]. However, a high proportion of cats are protected by vaccination, suggesting that immune mechanisms other than neutralizing antibodies may be involved. This conclusion was strengthened by results showing that a DNA vaccine engineered to promote the induction of FeLV-specific cytotoxic $\mathrm{T}$ cells provided protection against FeLV challenge without any detectable antibody responses $[19,20]$. However, it should not be concluded from these findings that neutralizing antibodies are not important. The presence of neutralizing antibodies in cats recovering from natural FeLV infection clearly correlates with a resistance to infection and the passive transfer of antibodies, either naturally through the colostrum [21] or experimentally by infusion [22], protecting cats against FeLV challenge.

Data presented here clearly show that immunization with Leucogen alone (group 2) was able to induce neutralizing antibodies (fig. 3a). This also indicates that our neutralization assay based on the measurement of provirus integration by real-time PCR is highly sensitive to such antibodies.

Numerous attempts have been undertaken to improve the efficacy of vaccination. For example, immunization with immunostimulating complexes (ISCOM) containing the gp70/gp85 precursor molecules not only induced virus neutralizing antibodies, but also protection against infection [23]. The development of antibodies to gp70 and p15E was confirmed by Western blot [24]. When compared with the commercial vaccine Leucocell, containing inactivated $\mathrm{FeLV}$, the ISCOM preparation proved to be superior. Virus-neutralizing antibodies were also induced by synthetic peptides corresponding to a domain of gp70 of FeLV-A involved in infection [25]. Since FeLV$\mathrm{B}$ and $\mathrm{FeLV}-\mathrm{C}$ might originate from a recombination between FeLV-A and endogenous FeLV-related sequences [26], neutralizing antibodies against FeLV-A should also protect cats from natural infection with all subgroups. The use of the transmembrane envelope protein described here is a new approach to induce broadly neutralizing antibodies which has been shown to be successful in the case of PERV [12], KoRV [15], and FeLV in different animals including cats $[13,14]$.

In previous immunization experiments with p15E of FeLV-A, the induced antisera neutralized FeLV-A and 2-4 epitopes were identified in the ectodomain of FeLV-A
p15E $[13,14]$. These same epitopes were recognized when p15E was used for immunization together with Leucogen (fig. 4c). Two epitopes, designated Ela (LETAQFRQL) and E1b (IQALEESISALEK), were localized in the FPPR; the other 2, E2a (KQRQQL) and E2b (WFEGWFN) (amino acids identical with an epitope recognized by antibodies broadly neutralizing HIV-1 are in bold), were localized in the MPER. It is important to underline that epitopes E2a and E2b correspond in their localization to the epitopes of 2F5 and 4E10, 2 monoclonal antibodies generated from HIV-1-infected individuals, broadly neutralizing HIV-1 and that there is - despite the evolutionary distance between HIV-1 and gammaretroviruses such as FeLV, KoRV, and PERV - a sequence homology of at least 3 amino acids. The epitope of the antibody $4 \mathrm{E} 10$ broadly neutralizing HIV-1 is NWFNIT (identical amino acids in bold) [5]. There is evidence that recognition of the epitopes E1b and E2b is crucial for neutralization (fig. 2) and that simultaneous immunization with $\mathrm{p} 15 \mathrm{E}$ and Leucogen changed the pattern of recognition by increasing the number of sera binding to Ela and E1b. It may be speculated that during immunization an interaction between both proteins occurs altering the conformation of the proteins. The E2b epitope also seems important for neutralization since the only serum not recognizing E2b (from animal 3.7) showed a significantly reduced neutralization titer against FeLV (fig. 4a, b).

Despite the similar localization and the limited sequence homology of these epitopes, neutralizing antisera against FeLV do not neutralize HIV (data not shown). In parallel, immunization with recombinant gp41 or epitopes recognized by broadly neutralizing antibodies such as 2F5 and 4E10 did not induce antibodies neutralizing HIV-1.

The results presented here show that immunizations with both the surface and the transmembrane envelope proteins (p15E and p45) induced higher titers of neutralizing antibodies suggesting that this combination may be the vaccination strategy of the future. This opinion is strengthened by the results of immunization studies using both envelope proteins of the murine leukemia virus $[27,28]$. 


\section{References}

$\checkmark 1$ Arita I: Farewell to smallpox vaccination. Dev Biol Stand 1979;43:283-296.

-2 McGaughey GB, Citron M, Danzeisen RC, Freidinger RM, Garsky VM, Hurni WM, Joyce JG, Liang X, Miller M, Shiver J, Bogusky MJ: HIV-1 vaccine development: constrained peptide immunogens show improved binding to the anti-HIV-1 gp41 MAb. Biochemistry 2003;25:3214-3223.

-3 Armbruster C, Stiegler GM, Vcelar BA, Jager W, Koller U, Jilch, R Ammann CG, Pruenster M, Stoiber H, Katinger HW: Passive immunization with the anti-HIV-1 human monoclonal antibody (hMAb) 4E10 and the hMAb combination 4E10/2F5/2G12. J Antimicrob Chemother 2004:54:915-920.

-4 Muster T, Steindl F, Purtscher M, Trkola A, Klima A, Himmler G, Ruker F, Katinger H: A conserved neutralizing epitope on gp41 of human immunodeficiency virus type 1 . J Virol 1993;67:6642-6647.

$\checkmark 5$ Kunert R, Wolbank S, Stiegler G, Weik R, Katinger H: Characterization of molecular features, antigen-binding, and in vitro properties of IgG and IgM variants of 4E10, an anti-HIV type 1 neutralizing monoclonal antibody. AIDS Res Hum Retroviruses 2004; 20:755-762.

-6 McGaughey GB, Barbato G, Bianchi E, Freidinger RM, Garsky VM, Hurni WM, Joyce JG, Liang X, Miller MD, Pessi A, Shiver JW, Bogusky MJ: Progress towards the development of a HIV-1 gp41-directed vaccine. Curr HIV Res 2004;2:193-204.

7 Zhang H, Huang Y, Fayad R, Spear GT, Qiao $\mathrm{L}$ : Induction of mucosal and systemic neutralizing antibodies against human immunodeficiency virus type 1 (HIV-1) by oral immunization with bovine papillomavirusHIV-1 gp41 chimeric virus-like particles. J Virol 2004;78:8342-8348.

8 Jarrett WF: The epidemiology of feline leukemia virus infection. Bibl Haematol 1975; 43:209-211.

\ Jarrett O, Hardy WD, Golder MC, Hay D: The frequency of occurrence of feline leukemia virus subgroups in cats. Int J Cancer 1978;21:334-337.
10 Sparkes AH: Feline leukemia virus: a review of immunity and vaccination. J Small Anim Pract 1997;38:187-194.

11 Jarrett O, Ganiere JP: Comparative studies of the efficacy of a recombinant feline leukaemia virus vaccine. Vet Rec 1996;138:7-11.

12 Fiebig U, Stephan O, Kurth R, Denner J: Neutralizing antibodies against conserved domains of p15E of porcine endogenous retroviruses: basis for a vaccine for xenotransplantation? Virology 2003;307:406-413.

13 Langhammer S, Fiebig U, Kurth R, Denner J: Neutralising antibodies against the transmembrane protein of feline leukaemia virus (FeLV). Vaccine 2005;23:3341-3348.

14 Langhammer S, Hübner J, Kurth R, Denner J: Antibodies neutralising feline leukaemia virus $(\mathrm{FeLV})$ in cats immunised with the transmembrane envelope protein p15E. Immunology 2006;117:229-237.

-15 Fiebig U, Hartmann MG, Bannert N, Kurth R, Denner J: Transspecies transmission of the endogenous koala retrovirus (KoRV). J Virol 2006;80:5651-5654.

16 Follis KE, Larson SJ, Lu M, Nunberg JH: Genetic evidence that interhelical packing interactions in the gp41 core are critical for transition of the human immunodeficiency virus type 1 envelope glycoprotein to the $\mathrm{fu}$ sion-active state. J Virol 2002;76:7356-7362.

17 Hofmann-Lehmann R, Tandon R, Boretti FS, Meli ML, Willi B, Cattori V, GomesKeller MA, Ossent P, Golder MC, Flynn JN, Lutz H: Reassessment of feline leukaemia virus (FeLV) vaccines with novel sensitive molecular assays. Vaccine 2006;24:1087-1094.

18 Jarrett O: Feline leukaemia virus neutralising antibodies. Vet Rec 2001;17:632.

19 Hanlon L, Argyle D, Bain D, Nicolson L, Dunham S, Golder MC, McDonald M, McGillivray C, Jarrett O, Neil JC, Onions DE: Feline leukemia virus DNA vaccine efficacy is enhanced by coadministration with interleukin-12 (IL-12) and IL-18 expression vectors. J Virol 2001;75:8424-8433.
20 Flynn JN, Hanlon L, Jarrett O: Feline leukaemia virus: protective immunity is mediated by virus-specific cytotoxic $\mathrm{T}$ lymphocytes. Immunology 2000;101:120-125.

21 Hoover EA, Olsen RG Jr, Hardy WD, Schaller JP: Horizontal transmission of feline leukemia virus under experimental conditions. J Natl Cancer Inst 1977;58:443-444.

22 Haley PJ, Hoover EA, Quackenbush SL, Gasper PW, Macy DW: Influence of antibody infusion on pathogenesis of experimental feline leukemia virus infection. J Natl Cancer Inst 1985;74:821-827.

23 Osterhaus A, Weijer K, Uytdehaag F, Jarrett $\mathrm{O}$, Sundquist B, Morein B: Induction of protective immune response in cats by vaccination with feline leukemia virus iscom. J Immunol 1985;135:591-596.

24 Osterhaus A, Weijer K, UytdeHaag F, Knell P, Jarrett O, Akerblom L, Morein B: Serological responses in cats vaccinated with FeLV ISCOM and an inactivated FeLV vaccine. Vaccine 1989;7:137-141.

25 Weijer K, Pfauth A, van Herwijnen R, Jarrett O, Meloen RH, Tomee C, Osterhaus AD: Induction of feline leukaemia virus-neutralizing antibodies by immunization with synthetic peptides derived from the FeLV env gene. Vaccine 1993;11:946-956.

26 Bechtel MK, Mathes LE, Hayes KA, Phipps AJ, Roy-Burman P: In vivo evolution and selection of recombinant feline leukemia virus species. Virus Res 1998;54:71-86.

27 Kleiser C, Schneider J, Bayer H, Hunsmann G: Immunoprevention of Friend leukaemia virus-induced erythroleukaemia by vaccination with aggregated gp70. J Gen Virol 1986; 67:1901-1907.

28 Thiel HJ, Schwarz H, Fischinger P, Bolognesi D, Schäfer W: Role of antibodies to murine leukemia virus p15E transmembrane protein in immunotherapy against AKR leukemia: a model for studies in human acquired immunodeficiency syndrome. Proc Natl Acad Sci USA 1987;84:5893-5897. 